\title{
First-Principles Study on Electronic Structures of Rich-Ni Al-based Quasicrystals Cluster
}

\author{
Yuan-Zhuo ZHANG ${ }^{1,2}$, Shu-Peng SONG ${ }^{1,2, *}$, He-Rong ZHOU ${ }^{1}$, Tian WAN ${ }^{1}$, \\ Pei-Yi LI ${ }^{1,2}$, Run WU', \\ ${ }^{1}$ State Key Laboratory of Refractories and Metallurgy, Wuhan University of Science and \\ Technology, Wuhan, 430081, People's Republic of China \\ ${ }^{2}$ Department of Materials and Metallurgy, Wuhan University of Science and Technology, Wuhan, \\ 430081, People's Republic of China \\ *Corresponding author: spsong@wust.edu.cn
}

Keywords: Quasicrystals, Cluster Model, First-Principles Calculation, Surface Energy

\begin{abstract}
The geometrical structure, electronic properties, surface energy and formation enthalpy of Ni-rich Al-based quasicrystals cluster models have been studied with the generalized gradient approximation (GGA) based on the density functional theory (DFT). Based on the first-principles calculation method, the ground state structures of $5 \mathrm{f}$ and $10 \mathrm{f}$ clusters are changed obviously due to atomic position space occupy around cluster center and their symmetry. The results from calculation demonstrate that the $10 \mathrm{f}$ model has smaller energy gap.
\end{abstract}

\section{Introduction}

Since the discovery of quasicrystalline I-phase in Al-Mn alloys, two dimensional quasicrystal phase aslo was investigated in Al-Co-Ni alloy [1]. For unit cell of Al-Co-Ni quasicrystal, the diameter of 10-fold and 5-fold symmetric pentagonal columnar clusters are $2 \mathrm{~nm}$, for basic Ni-rich structure the gap of layer is $0.2 \mathrm{~nm}$. There exists short-range chemical order inside clusters, which means the first-principles calculation could be employed to study its structural properties [2,3,4].

The physical and chemical properties of quasicrystal have been studied in experiments by scholars, but the electronic properties of quasicrystals are rarely considered [5]. Especially for thermally stable Al-Ni-Co quasicrystal system, first-principles calculations can provide important insights to the understanding of quasi-periodic clusters [6,7].

In this paper, the structure, energy, geometry optimization, electronic density of two dimensional Al-Ni-Co quasicrystals were investigated. There were two cluster models of this quasicrystal, one is 5 -fold symmetry (5f) cluster and the other is 10 -fold symmetry (10f) cluster. By comparing the energy of the two models, we found Ni-rich $10 \mathrm{f}$ cluster model were more stable. The displacement of the ten atoms in the center implied the position change caused by thermodynamic conditions. The electron density shows an internal perspective to explore the particular physical and chemical properties of Al-Ni-Co quasicrystals.

\section{Models and Calculations}

In this study, there are two models which are Ni-rich $10 \mathrm{f}$ and Ni-rich $5 \mathrm{f}$ models, they both have two layers as showed in Fig. 1, while the distance of the two layers is $0.2 \mathrm{~nm}$, and the diameter of a columnar cluster is $2 \mathrm{~nm}$. Hypothetical lattice constants are $a=b=3 \mathrm{~nm}, c=1.2 \mathrm{~nm}, \alpha=\beta=\gamma=90$, which could assure that calculations of the cluster would not be affected by their nearby clusters. For Ni-rich Al-Co-Ni quasicrystal, Ni-potential was employed to simplify the calculations. The chemical composition of both Ni-rich $10 \mathrm{f}$ and Ni-rich $5 \mathrm{f}$ models are $\mathrm{Al}_{55} \mathrm{Ni}_{35}$. The orange atoms are $\mathrm{Al}$, while the other blue ones are $\mathrm{Ni}$.

The CASTEP [8], based on the first principles density functional theory, within the GGA was used to perform the calculations. For the GGA exchange-correlation, the Perdew-Burke-Ernzerhof [9] was employed. 

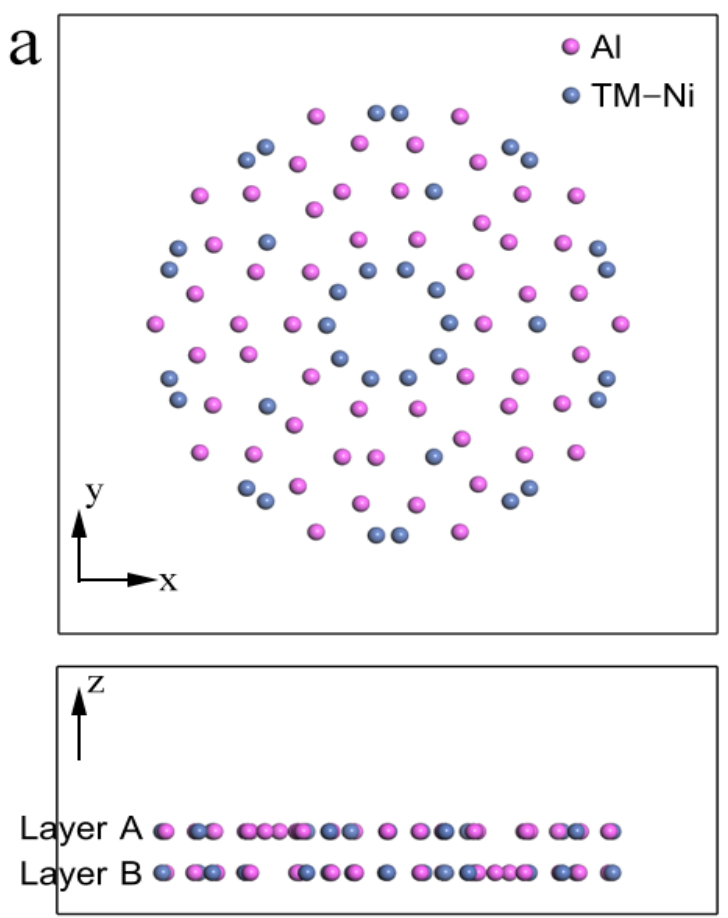
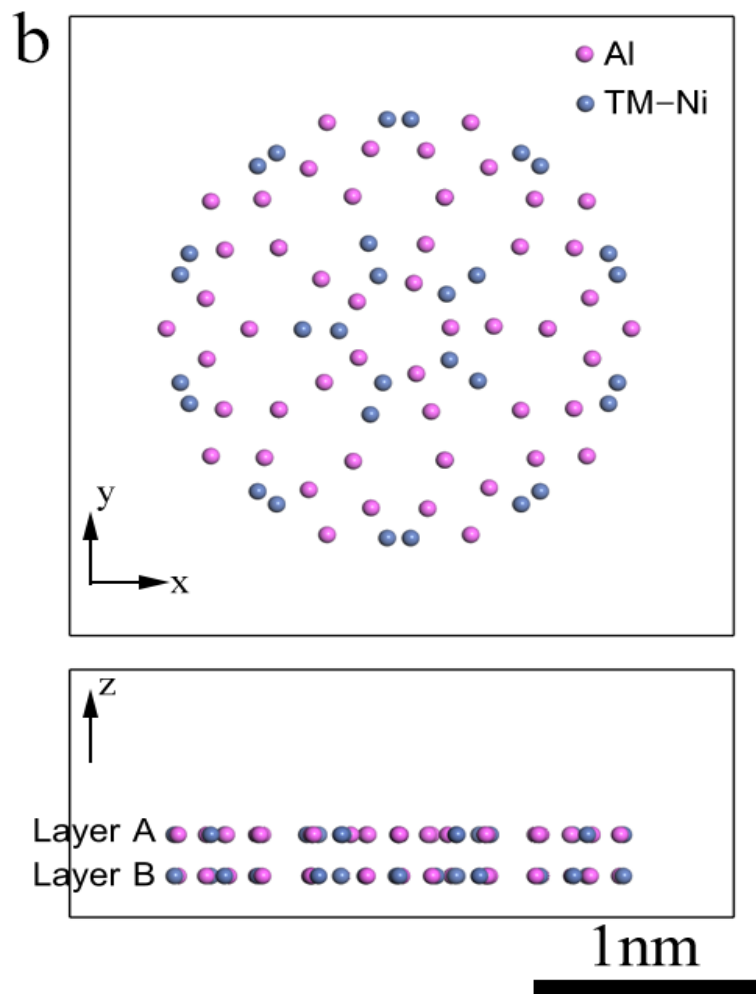

Fig. 1. Atomic cluster models of Ni-rich $10 \mathrm{f}(\mathrm{a})$ and Ni-rich $5 \mathrm{f}(\mathrm{b})$

Ultrasoft pseudo-potential and plane wave basis sets were applied [10]. The cutoff energy was tested from $300 \mathrm{eV}$ to $500 \mathrm{eV}$, but the difference of ground state energy was less than $0.1 \%$. So the cutoff energy was set at $300 \mathrm{eV}$ to improve efficiency. The $k$-points were tested from $1 \times 1 \times 1$ to $1 \times 1 \times 3$, the difference of the ground state energy was also less than $0.1 \%$, so the $k$-points were set at $1 \times 1 \times 1$. And the self-consistent convergence of the total energy was lower than $1 \times 10^{-6} \mathrm{eV} / \mathrm{atom}$. The geometry optimization was used the BFGS method. Forces on atoms were calculated through the Hellmann-Feynman theory as the partial derivatives of the free energy with respect to the atomic position. And the force was smaler than $0.03 \mathrm{eV} / \AA$.

\section{Results and Discussion}

\subsection{Geometry Optimization}

According to four layers $\mathrm{Al}_{110} \mathrm{Ni}_{70}$ model (ABA'B') calculation results, when all atoms of $5 \mathrm{f}$ and $10 \mathrm{f}$ models were constrained except the center ten atoms in the layer B and A', it was found that some displacement of the ten atoms seemed to be consistent with the thermal motion.

Table 1 Position changes of central atoms in Ni-rich 10f model after geometry optimization. ( $\AA$ )

\begin{tabular}{cccccccc}
\hline Coordinates & \multirow{2}{*}{$\begin{array}{c}\text { Cor } \\
\text { Atoms }\end{array}$} & & $y$ & \multirow{2}{*}{$x^{\prime}$} & $y^{\prime}$ & $z^{\prime}$ & $d$ \\
\hline Ni1 & 12.720 & 16.560 & 4.000 & 12.604 & 16.672 & 4.085 & 0.182 \\
Ni2 & 15.801 & 17.637 & 4.000 & 15.835 & 17.672 & 4.065 & 0.081 \\
Ni3 & 17.790 & 15.060 & 4.000 & 17.830 & 15.025 & 4.037 & 0.064 \\
Ni4 & 15.918 & 12.405 & 4.000 & 15.943 & 12.263 & 4.074 & 0.162 \\
Ni5 & 12.801 & 13.332 & 4.000 & 12.658 & 13.243 & 4.092 & 0.192 \\
Ni6 & 17.286 & 13.440 & 2.000 & 17.300 & 13.362 & 1.968 & 0.086 \\
Ni7 & 14.202 & 12.360 & 2.000 & 14.162 & 12.346 & 1.992 & 0.043 \\
Ni8 & 12.216 & 14.940 & 2.000 & 12.195 & 14.960 & 1.996 & 0.030 \\
Ni9 & 14.085 & 17.595 & 2.000 & 14.087 & 17.633 & 1.992 & 0.039 \\
Ni10 & 17.205 & 16.659 & 2.000 & 17.269 & 16.692 & 1.965 & 0.080 \\
\hline
\end{tabular}


Specific changes has taken place in the geometry optimization which were shown as Table 1 and Table 2, it should be noted that the displacement $(d)$ are less than $0.2 \AA$, this phenomenon was similar to the experinental thermal motion of Al-Ni-Co quasicrystals.

Table 2 Position changes of central atoms in Ni-rich $5 f$ model after geometry optimization. ( $\AA$ )

\begin{tabular}{cccccccc}
\hline $\begin{array}{c}\text { Coordinates } \\
\text { Atoms }\end{array}$ & $x$ & $y$ & $z$ & $x^{\prime}$ & $y^{\prime}$ & $z^{\prime}$ & $d$ \\
\hline Al1 & 12.981 & 16.178 & 4.000 & 12.924 & 16.200 & 4.063 & 0.088 \\
Al2 & 13.049 & 13.464 & 4.000 & 13.015 & 13.421 & 4.062 & 0.083 \\
Al3 & 15.656 & 12.688 & 4.000 & 15.684 & 12.647 & 4.062 & 0.079 \\
Al4 & 17.202 & 14.919 & 4.000 & 17.256 & 14.939 & 4.063 & 0.085 \\
Al5 & 15.547 & 17.078 & 4.000 & 15.545 & 17.131 & 4.062 & 0.082 \\
Ni1 & 12.161 & 14.766 & 2.000 & 12.221 & 14.799 & 2.066 & 0.095 \\
Ni2 & 14.151 & 12.246 & 2.000 & 14.129 & 12.320 & 2.066 & 0.102 \\
Ni3 & 17.153 & 13.354 & 2.000 & 17.080 & 13.365 & 2.069 & 0.101 \\
Ni4 & 17.029 & 16.546 & 2.000 & 16.995 & 16.481 & 2.066 & 0.099 \\
Ni5 & 13.943 & 17.425 & 2.000 & 13.989 & 17.368 & 2.067 & 0.099 \\
\hline
\end{tabular}

After relax, in 10f model, the center atoms of layer B and A' are moving closer to layer A and B', with $u_{\mathrm{B}}=0.071 \AA$ and $u_{\mathrm{A}^{\prime}}=-0.018 \AA$. In the $5 \mathrm{f}$ model, after relax, the five $\mathrm{Al}$ atoms of layer $\mathrm{B}$ are closer to layer $\mathrm{A}\left(u_{\mathrm{B}}=0.062 \AA\right)$, and the layer $\mathrm{A}^{\prime}$ five Ni atoms are moving far away from layer B' $\left(u_{\mathrm{A}^{\prime}}\right.$ $=0.067 \AA$ ). It implies that higher temperature variation lead to a active displacement in the center of quasicrsytal clusters.

\subsection{Ground State Energy}

The bulk stability of the compound is determined by formation enthalpy. The negative formation enthalpy change means the structure is thermodynamically stable and the positive value implies the structure is instable [11]. The bulk energy is calculated by using the following expression:

$$
E_{\text {bulk }}=\left(E_{a}-E_{b}\right) /(a-b)
$$

Where $E_{a}$ is the ground state energy of four-layer quasicrystal, and $E_{b}$ is the ground state energy of two layers. By Eq. 1, the ground state energy of single layer, which is the bulk energy, can be calculated. With the bulk energy, the surface energy is calculated as following expression [12]:

$$
E_{\text {surf }}=\left(E_{\text {slab }}-n \cdot E_{\text {bulk }}\right) / 2 A
$$

$A$ is the area of the columnar clusters' top surface, $E_{\text {slab }}$ is total energy of a slab with n layers. After solving Eq. 2, the formation energy is defined as:

$$
\Delta H_{f}=\left[\left(2 E_{\text {bulk }}-\sum\left(n_{i} \cdot E_{i}\right)\right] / \mathrm{n}\right.
$$

$E_{i}$ is the energy per atom of bulk system, $n_{i}$ represent the number of the reference atom in the layers. $\mathrm{n}$ is the total number of the atoms. The formation enthalpy is summarized in table 3 by Eq. 3 . The results of $\Delta H_{f}$ are negative and clearly verify the stability of the studied compounds.

Table 3 Calculated ground state energies (eV/atom), surface energy (eV/nm²) and formation enthalpy (eV/atom)

\begin{tabular}{cccc}
\hline Symmetry & $E(\mathrm{eV} / \mathrm{atom})$ & $E_{\text {surf }}\left(\mathrm{eV} / \mathrm{nm}^{2}\right)$ & $\Delta \mathrm{H}_{f}(\mathrm{eV} / \mathrm{atom})$ \\
\hline $10 \mathrm{f}$ & -541.986 & 10.762 & -4.770 \\
$5 \mathrm{f}$ & -541.809 & 10.318 & -4.563 \\
\hline
\end{tabular}

\subsection{Electron Density}

Electron density has been calculated to study the nature of the bonds. Fig. 2(a)-(c) are layer A and B of Ni-rich 10f model, Fig. 2(b)-(d) are layer A and B of $5 f$ model. Black contrast distribution represents higher charge density while gray areas represents lower. 


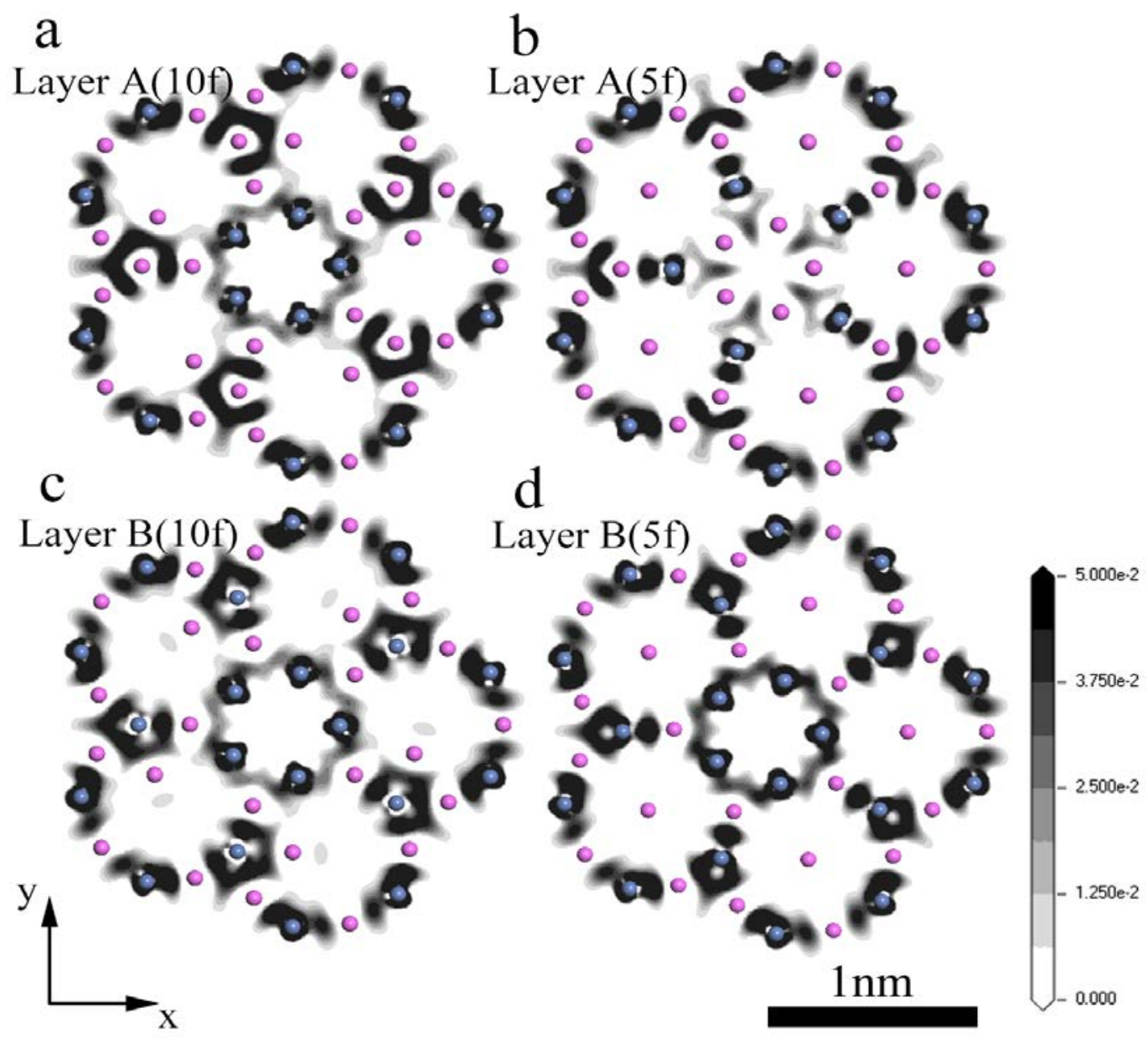

Fig. 2. Electron density of layer A and layer B of Ni-rich $10 \mathrm{f}(\mathrm{a}, \mathrm{c})$ and Ni-rich $5 \mathrm{f}(\mathrm{b}, \mathrm{d})$ models

Partial enlarged images of electron density maps of Ni-rich quasicrystal model are shown as Fig. 3 and Fig. 4, which clearly indicates the cluster central electronic compositions of band structures.

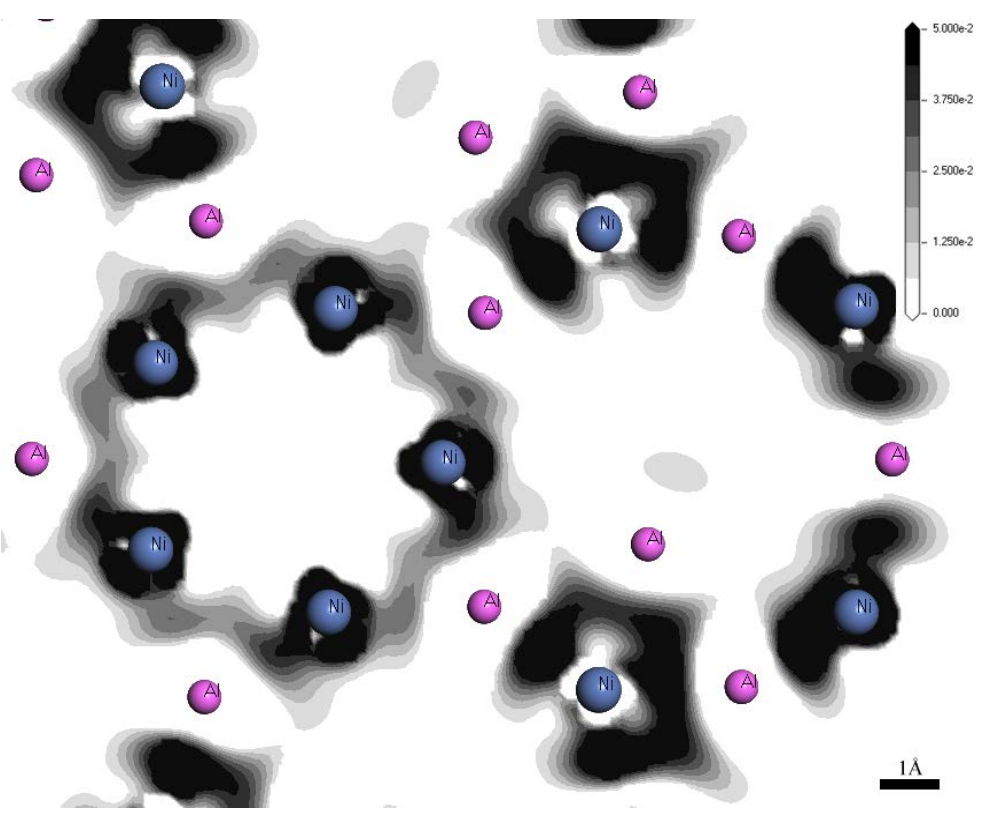

Fig. 3. Partial electron density of layer B of Ni-rich $10 \mathrm{f}$ model 


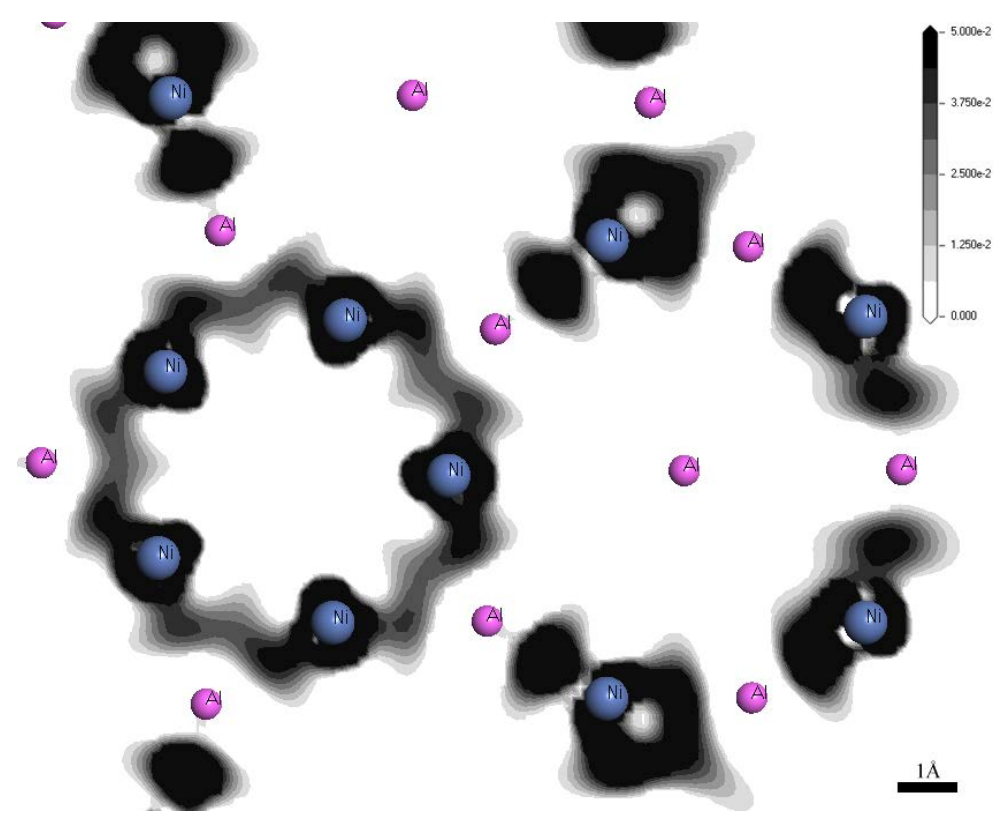

Fig. 4. Partial electron density of layer B of Ni-rich $5 \mathrm{f}$ model

In general, with higher value of electronegativity 1.88, the $\mathrm{Ni}$ atom have more powerful ability to attract electrons from near $\mathrm{Al}$ atoms (electronegativity is 1.61). So, there are higher density of electron clouds around $\mathrm{Ni}$ atoms. in Fig. 4, the electron clouds around the central five $\mathrm{Ni}$ atoms is different, while same area have deeper electron density contrast in Fig. 4. The differece means the tighter combination of central five $\mathrm{Ni}$ atoms, which probably leads to low coherence with nearby $\mathrm{Al}$ atoms, then the stability of model is worse, just as shown in the energy analysis.

\section{Summary}

We performed first principles calculations to investigate the geometrical structure and electronic structures of Ni-rich $5 \mathrm{f}$ and $10 \mathrm{f}$ Al-based quascrystal cluster models. The surface energy and formation enthalpy was also calculated to establish linkage between the local chemical ordering and atomic displacement.

\section{Acknowledgements}

This work is financially co-supported by National Nature Science Foundation of China (50901053), Nature Science Foundation of Hubei Province (2014CFB799), Foundation of State Key Laboratory of Refractories and Metallurgy (2014QN19).

\section{References}

[1] Shechtman D, Blech I, Gratias D, et al. Metalisc phase with long-range orientational order and no translational symmetry [J]. Physical Review Letters, 1984, 53: 1951-1954.

[2] Ritsch S, Beeli C, Nissen $\mathrm{H}$ U, et al. The existence regions of structural modifications in decagonal Al-Co-Ni[J]. Philosophical magazine letters, 1998, 78(2): 67-75.

[3] Saitoh K, Tsuda K, Tanaka M, et al. Structural Study of an Al72Ni20Co8 Decagonal Quasicrystal Using the High-Angle Annular Dark-Field Method.[J]. Japanese Journal of Applied Physics, 1997, 36(36):L1400-L1402.

[4] Abe $\mathrm{H}$, Saitoh $\mathrm{H}$, Kusawake T, et al. An order-disorder transformation in $\mathrm{Al}_{72} \mathrm{Ni}_{20} \mathrm{Co}_{8}$ decagonal quasicrystals[J]. Journal of alloys and compounds, 2002, 342(1): 241-245. 
[5] Ritsch S, Beeli C, Nissen H U, et al. Two different superstructures of the decagonal Al-Co-Ni quasicrystal[J]. Philosophical Magazine A, 1995, 71(3): 671-685.

[6] Nejadsattari F, Stadnik Z M, Przewoźnik J, et al. Mössbauer spectroscopy, magnetic, and ab-initio study of the approximant $\mathrm{Al}_{76} \mathrm{Ni}_{9} \mathrm{Fe}_{15}$ to a decagonal Al-Ni-Fe quasicrystal[J]. Journal of Alloys and Compounds, 2016, 662: 612-620.

[7] Edagawa K, Ichihara M, Suzuki K, et al. New type of decagonal quasicrystal with superlattice order in Al-Ni-Co alloy[J]. Philosophical magazine letters, 1992, 66(1): 19-25.

[8] Segall M D, Lindan P J D, Probert M J, et al. First-principles simulation: ideas, illustrations and the CASTEP code[J]. Journal of Physics: Condensed Matter, 2002, 14(11): 2717-2744.

[9] Perdew J P, Burke K, Ernzerhof M. ERRATA:Generalized gradient approximation made simple[J]. Physical Review Letters, 1996, 77(18):3865-3868.

[10] Vanderbilt D. Soft self-consistent pseudopotentials in a generalized eigenvalue formalism[J]. Physical Review B, 1990, 41(11):7892-7895.

[11] Cao Y, Zhu P, Zhu J, et al. First-principles study of NiAl alloyed with Co[J]. Computational Materials Science, 2016, 111: 34-40.

[12] Wang F, Cao K, Wu Y, et al. Interfacial properties of the enhanced visible-light plasmonic Ag/Bi2WO 6 (0 0 1) nanocomposite[J]. Applied Surface Science, 2016, 360: 1075-1079. 